\title{
Determination of the Diffusion Coefficient of Insulin and Lysozyme in Crosslinked Dextran Hydrogels by Pulsed-Field-Gradient NMR
}

\author{
Yukio Aso,* Sumie Yoshioka, and Shigeo Kojıma \\ National Institute of Health Sciences, 1-18-1 Kamiyoga, Setagaya-ku, Tokyo 158-8501, Japan. \\ Received June 1, 1998; accepted August 6, 1998
}

Crosslinked dextran hydrogels were prepared from glycidyl methacrylate (GMA)-derivatized dextran by gamma-irradiation initiation in the presence of insulin and lysozyme as model protein and peptide drugs. The diffusion coefficient of insulin and lysozyme in the hydrogels was determined successfully by a pulsed-field-gradient stimulated-echo method. A conventional NMR probe which can impose a relatively small magnetic field gradient $(30 \mathrm{Gauss} / \mathrm{cm})$ was used for the measurements. This method seems to be applicable to proteins and peptides with a molecular weight of about 10000 or less. The diffusion coefficient determined by the NMR method almost agreed with that calculated from the drug release profiles, indicating the usefulness of the diffusion coefficient determined by the NMR method as a measure of the release rate from hydrogels.

Key words diffusion; hydrogels; lysozyme; insulin; drug release

Hydrogels are biocompatible materials and have attracted much attention as vehicles for protein and peptide drug delivery systems. ${ }^{1-2)}$ Measurement of drug diffusion in hydrogels is interesting because the diffusion coefficient is a direct measure of the rate of drug release from the hydrogels and gives information on the polymer network structure. There are many methods for measuring the diffusion coefficient of drugs in gels, ${ }^{3)}$ including (1) methods for measuring drug flux through a gel diaphragm, (2) methods for measuring the time-profile of drug release from gels, and (3) instrumental methods such as dynamic light scattering, fluorescence recovery after photobleaching, and the pulsed-field-gradient spin-echo (PFG-SE) method. From the viewpoint of total experimental time, instrumental methods are superior to methods for measuring drug flux or drug release. Since instrumental methods are non-invasive, the measured diffusion coefficient can be confirmed by other methods determining the drug release rate. The PFG-SE method, in particular, has the advantage of accuracy and precision in comparison with the other instrumental methods, ${ }^{3)}$ and has been used to determine the diffusion coefficient of small molecules. For example, the release rates of adinazolam methylate from extended-release dosage forms of a hydroxypropylmethylcellulose matrix have been predicted from the diffusion coefficient of the drug in the hydrated matrices (hydrogels) by this method. ${ }^{4)}$ The diffusion coefficient of water molecules in poly(2-hydroxyethylmethacrylate) hydrogels and silk protein gels has also been reported. ${ }^{5,6)}$ Although the diffusion coefficient of peptides and proteins in solutions has been measured by the PFGNMR method to characterize protein unfolding and the selfassociation of peptides and proteins, ${ }^{7-10)}$ few studies have focused on the determination of the diffusion coefficient of peptides and proteins in hydrogels.

The diffusion coefficient of protein in hydrogels cannot be measured accurately by the PFG-SE method using a conventional high-resolution NMR probe which can impose only a small magnetic field gradient (30 Gauss $/ \mathrm{cm}$ ). In the PFG-SE method (Fig. 1(a)), the magnetization of the sample stays in the $x y$ plane the during diffusion period $(\Delta)$, and the echo intensity is governed by the spin-spin relaxation rate $\left(R_{2}\right)$ and the diffusion coefficient $(D)$ of the sample as described by Eq. 1.

* To whom correspondence should be addressed.

$$
\begin{aligned}
A(2 \tau)= & A(0) \exp \left(-2 \tau R_{2}\right) \times \exp \left\{-(\gamma G \delta)^{2}(\Delta-\delta / 3) D-2\left(\gamma G_{0}\right)^{2} D \tau^{3} / 3\right\} \\
& \times \exp \left\{\gamma^{2} G G_{0} \delta D\left(t_{1}{ }^{2}+t_{2}{ }^{2}+\delta\left(t_{1}+t_{2}\right)+2 \delta^{2} / 3-2 t^{2}\right)\right\}
\end{aligned}
$$

where $A(2 \tau)$ is the echo intensity at time $2 \tau$ and $A(0)$ is initial signal intensity. $\gamma, G, \delta, \Delta$ and $G_{0}$ are the gyromagnetic ratio of the nuclei observed (proton), the intensity of the field gradient pulse, the duration of the gradient pulses, the interval of two gradient pulses and the steady-state field gradient, respectively. As indicated by Eq. 1, a large field gradient pulse is needed to obtain the echo attenuation due to the diffusion process when $R_{2}$ is large. Thus, the PFG-SE method with a small magnetic field gradient cannot determine the diffusion coefficient of protein in hydrogels since the $R_{2}$ of protein protons is very large due to their low mobility.
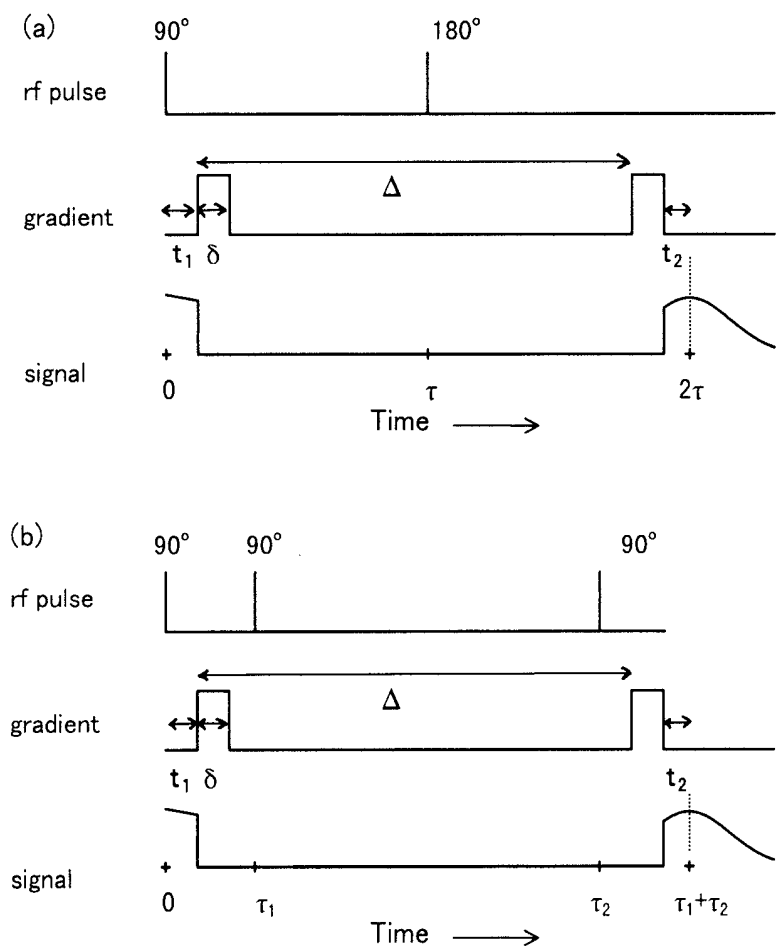

Fig. 1. Pulse Sequence of the Pulsed-Field-Gradient Spin-Echo Method (a) and Pulsed-Field-Gradient Stimulated-Echo Method (b)

(C) 1998 Pharmaceutical Society of Japan 
The stimulated-echo method (Fig. 1(b)), ${ }^{11)}$ which is a modified form of the PFG-SE method, seems to overcome the fast spin-spin relaxation of protein protons. In this method, the magnetization of the sample is stored along the $z$-axis during the diffusion period. The echo intensity depends on the spinlattice relaxation rate $\left(R_{1}\right), R_{2}$ and the $D$, as described by Eq. 2 . Since the $R_{1}$ of protein protons is much smaller than the $R_{2}$, the stimulated echo method can obtain the echo attenuation during a longer diffusion period than the PFG-SE method, and the echo attenuation by the spin-spin relaxation process can be minimized.

$$
\begin{aligned}
A\left(\tau_{1}+\tau_{2}\right)= & A(0) \exp \left\{-2 \tau_{1} R_{2}-\left(\tau_{2}-\tau_{1}\right) R_{1}\right\} \\
& \times \exp \left\{-(\gamma G \delta)^{2}(\Delta-\delta / 3) D-\left(\gamma G_{0}\right)^{2} D \tau_{1}{ }^{2}\left(\tau_{2}-\tau_{1} / 3\right)\right\} \\
& \times \exp \left\{\gamma^{2} G G_{0} \delta D\left(t_{1}{ }^{2}+t_{2}{ }^{2}+\delta\left(t_{1}+t_{2}\right)+2 \delta^{2} / 3-2 \tau_{1} \tau_{2}\right)\right\}
\end{aligned}
$$

In this study, we report the feasibility of the stimulatedecho method for determination of the diffusion coefficient of peptides and proteins in hydrogels using a conventional highresolution NMR probe. The correlation between the diffusion coefficient determined by NMR and that calculated from drug release profiles is also described.

\section{Materials and Methods}

Materials Dextran (average M.W. 40000), glycidyl methacrylate (GMA), 4-dimethylaminopyridine, insulin (from bovine pancreas) and lysozyme (from chicken egg white) and bovine serum albumin (BSA) were purchased from Wako Pure Chemical Industry, Ltd. (Osaka). Other chemicals used were of reagent grade. Methacrylated dextran was synthesized from dextran and GMA according to the method reported by Hennink et al ${ }^{121}$ The degree of GMA substitution (the number of GMA molecules per 100 glucose moieties) was determined to be $3-40 \%$ by ${ }^{~} \mathrm{H}$ nuclear magnetic resonance (NMR) (Unity plus 400, Varian).

Preparation of Hydrogels Lysozyme- or insulin-containing hydrogels were prepared in a manner similar to that reported previously. ${ }^{13.14)}$ Lysozyme (final concentration, $2 \%$ ) was dissolved in deuterium oxide and mixed with a deuterium oxide solution of methacrylated dextran (final concentration, $20 \%$ ). Insulin (final concentration, $2 \%$ ) was dissolved in deuterium oxide containing $2 \%$ deuterated acetic acid. The solutions were transferred to glass molds separated by 2 -mm-thick silicone rubber and irradiated with gamma rays at a dose of $1 \mathrm{kGy}$. The hydrogels were then cut into disks $(6 \mathrm{~mm}$ in diameter, for release studies) or strips $(15 \times 4 \times 2 \mathrm{~mm}$, for NMR studies).

Release Study Each hydrogel disk containing insulin or lysozyme was placed in a flat-bottomed vessel (internal diameter, $35 \mathrm{~mm}$ ) fitted with a screw cap, and $10 \mathrm{ml}$ phosphate buffer $(10 \mathrm{~mm}, \mathrm{pH} 7.4,0.9 \% \mathrm{NaCl}$ for lysozyme) or $2 \%$ acetic acid (for insulin) was added. The disk was maintained at 37 (for lysozyme) or $25^{\circ} \mathrm{C}$ (for insulin) with shaking at a rate of 110 strokes $/ \mathrm{min}$. At appropriate time intervals, $0.5 \mathrm{ml}$ solution was withdrawn and replaced by the same volume of fresh buffer solution. Drug released from the hydrogel was assayed by high performance liquid chromatography (HPLC). The HPLC conditions were: column, TSK gel phenyl $4.6 \mathrm{~mm} \times 75 \mathrm{~mm}$ (Tosoh, Tokyo); eluent, a linear gradient of $0-67 \%$ aqueous acetonitrile containing $0.05 \%$ trifluoroacetic acid; elution time, $20 \mathrm{~min}$; detection, $210 \mathrm{~nm}$.

NMR Measurement The diffusion coefficient of insulin and lysozyme in the dextran hydrogels was determined using a stimulated-echo pulse sequence ${ }^{11)}$ employing a UNITY plus spectrometer operating at $400 \mathrm{MHz}$ (Varian). Although the echo intensity depended in a complex manner on the spin-lattice relaxation rate, $R_{1}$, the spin-spin relaxation rate, $R_{2}$, and the diffusion coefficient, $D$, as shown in Eq. 2, the equation could be simplified by the delay times ( $\tau_{1}$ and $\tau_{2}$ in Eq. 2 ) being held constant, as below:

$$
A(G)=A_{0} \exp \left\{-(\gamma \delta)^{2}(\Delta-\delta / 3) G^{2} D\right\}
$$

where $A(G)$ and $A_{0}$ are the echo intensities, with and without the field gradient. A series of echoes were measured by systematically varying the intensity of the field gradient $(G)$ from 3 to 30 Gauss $/ \mathrm{cm}$. The echo intensity at each field gradient was obtained by integrating insulin or lysozyme signals in the aromatic region $(6.5-8 \mathrm{ppm})$ and the diffusion coefficient was calculated by fitting the integrated intensity data to Eq. 3 using a non-linear least squares program.

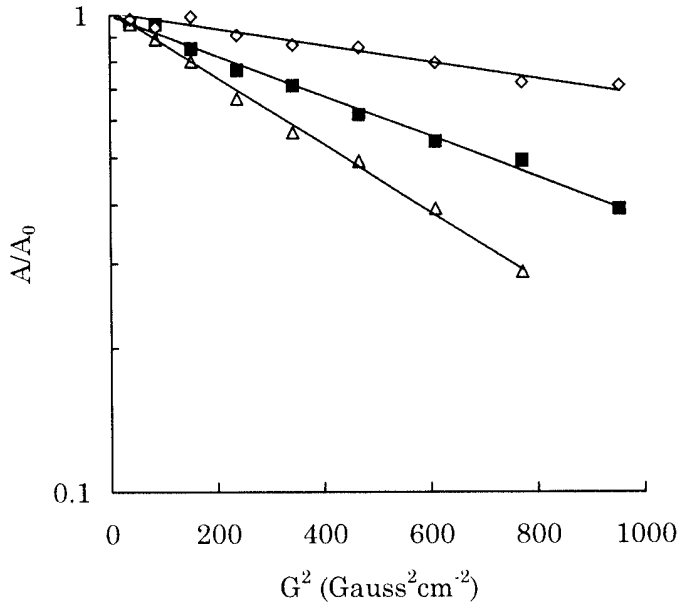

Fig. 2. Normalized Signal Intensity of Insulin in Dextran Hydrogels as a Function of the Square of the Field-Gradient Intensity $\left(G^{2}\right)$ at $25^{\circ} \mathrm{C}$

The signal intensity was obtained by integrating the insulin signals in the aromatic region $(6.6-8 \mathrm{ppm})$. Duration of gradient pulses $(\delta), 0.0075 \mathrm{~s}$; Interval between two gradient pulses $(\Delta), 0.11 \mathrm{~s} . \triangle$, in $6 \%$ dextran hydrogel; $\mathbf{\square}$, in $12 \%$ dextran hydrogel; $\diamond, 20 \%$ dextran hydrogels. The degree of GMA substitution is $16 \%$.

\section{Results and Discussion}

The diffusion coefficient of insulin and lysozyme in crosslinked dextran hydrogels could be successfully determined by the pulsed-field-gradient stimulated-echo method using a conventional NMR probe, which can impose a relatively small magnetic field gradient $(30 \mathrm{Gauss} / \mathrm{cm})$. Figure 2 shows the signal intensity of insulin (the integrated signals of aromatic protons, $6.5-8 \mathrm{ppm}$ ) in dextran hydrogels as a function of the square of the field-gradient intensity $\left(G^{2}\right)$. A linear relationship between the logarithm of the signal intensity and $G^{2}$ indicates that the diffusion coefficient of these proteins can be determined precisely with a maximum fieldgradient intensity of $30 \mathrm{Gauss} / \mathrm{cm}$. Similar results were obtained for lysozyme in dextran hydrogels. On the other hand, the diffusion coefficient of BSA in the hydrogels could not be determined because of the slower diffusion of BSA in the hydrogels and lower signal-to-noise ratio of the BSA signals compared with that of insulin or lysozyme. Larger field-gradient pulses may be needed for such samples with a slow diffusion rate. Figure 3 shows the diffusion coefficients of insulin and lysozyme in dextran hydrogels. The coefficients for both drugs decreased as the degree of GMA substitution increased (Fig. 3(a)). An increase of dextran concentration in the hydrogels resulted in a decrease in the diffusion coefficient of insulin (Fig. 3(b)). These results suggest that an increased degree of GMA substitution results in a decrease in the mesh size of the gel network, and that an increased dextran concentration leads to a decrease in the effective volume available for diffusion, both of which retard drug diffusion in the gels. ${ }^{12)}$

Figure 4 shows the release of insulin from the dextran hydrogels. The fraction of insulin released at time $t\left(M_{t} / M_{0}\right)$ conformed to the Higuchi equation (Eq. 4) ${ }^{15)}$ for values of $M_{t} / M_{0}$ less than 0.5 , indicating that drug release was governed by diffusion.

$$
M_{t} / M_{0}=k t^{1 / 2}
$$

where $k$ is the apparent release rate constant. Similar release profiles were observed for the hydrogels which con- 

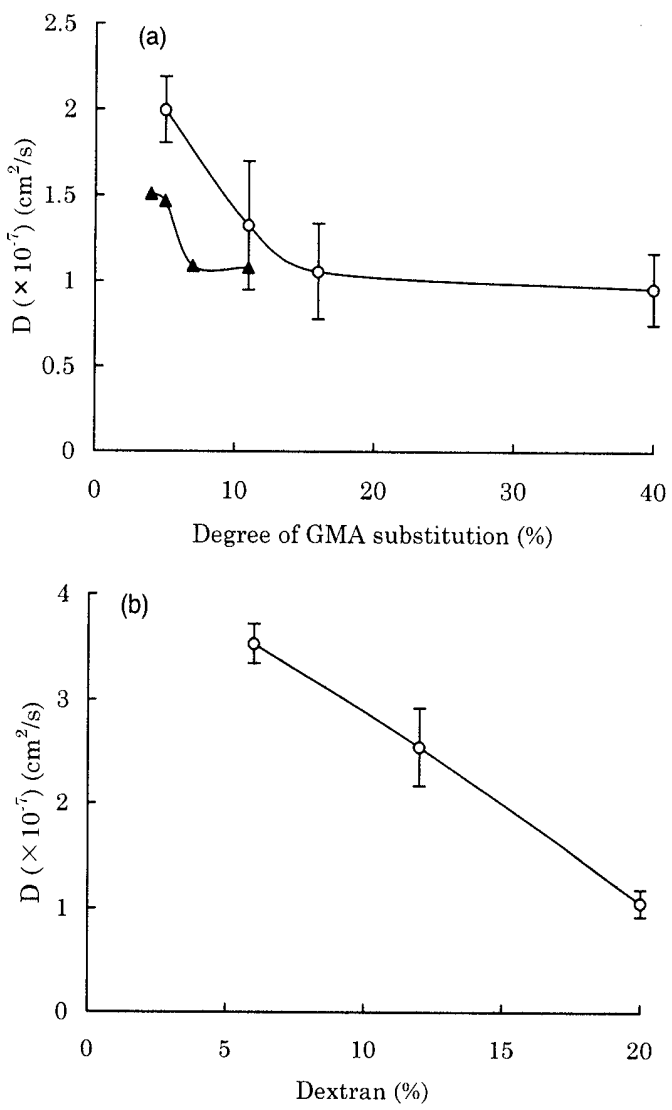

Fig. 3. Effect of the Degree of Crosslinking (a) and Dextran Concentration (b) on the Diffusion Coefficient of Insulin $(\bigcirc)$ and Lysozyme $(\mathbf{\Delta})$ in Dextran Hydrogels

(a): Dextran concentration was fixed at $20 \%$. The degree of crosslinking of dextran hydrogel was controlled by the degree of GMA substitution of dextran. (b): The degree of GMA substitution was fixed at $16 \%$. Measurement of the diffusion coefficient was carried out at $25^{\circ} \mathrm{C}$ for insulin and $37^{\circ} \mathrm{C}$ for lysozyme. The diffusion coefficients of insulin are the average of three determinations and the error bars represent the standard deviation. The diffusion coefficients of lysozyme are the average of two determinations.

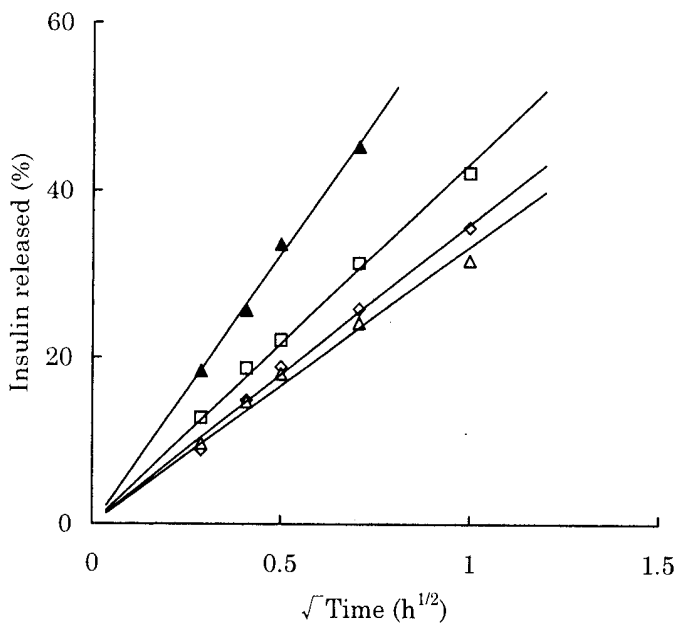

Fig. 4. Insulin Release from Dextran Hydrogels with Various Dextran Concentrations and Various Degrees of Crosslinking at $25^{\circ} \mathrm{C}$

$\Delta$ : Dextran concentration, 6\%; Degree of GMA substitution, $16 \%$. $\square$ : Dextran concentration, 20\%; Degree of GMA substitution, 11\%. $\diamond$ : Dextran concentration, $20 \%$; Degree of GMA substitution, $16 \%$. $\triangle$ : Dextran concentration, 20\%; Degree of GMA substitution, $40 \%$. The degree of crosslinking of dextran hydrogel was controlled by the degree of GMA substitution of dextran. The amount of insulin released was determined by HPLC.
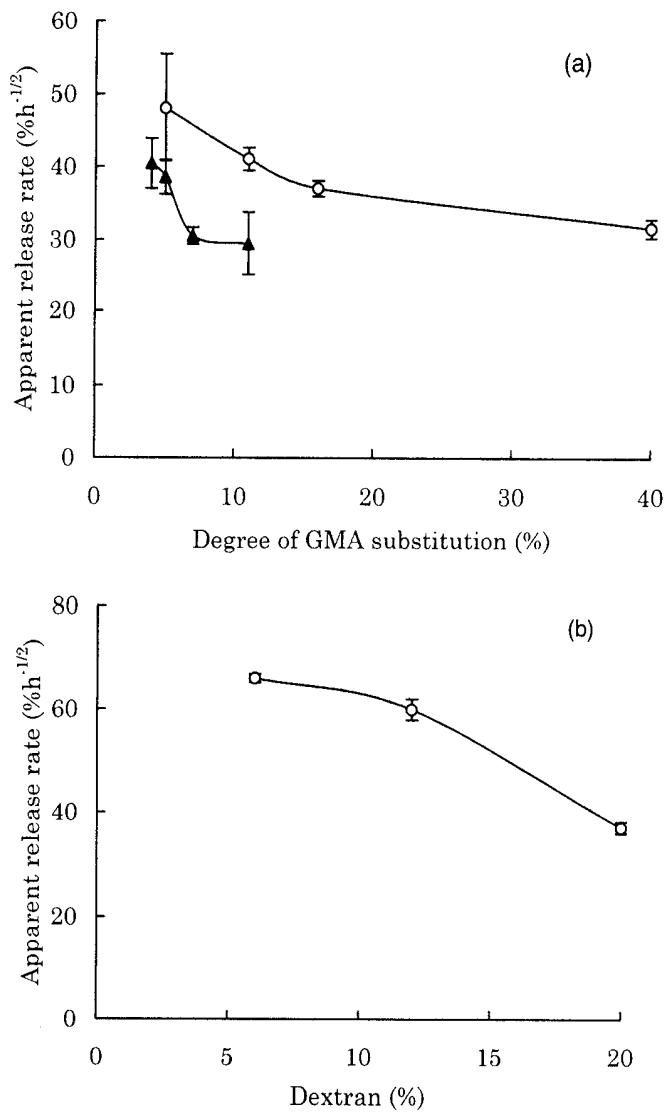

Fig. 5. Effect of the Degree of Crosslinking (a) and Dextran Concentration (b) on the Apparent Release Rate of Insulin $(O)$ and Lysozyme $(\boldsymbol{A})$ in Dextran Hydrogels

(a): Dextran concentration was fixed at $20 \%$. The degree of crosslinking of dextran hydrogel was controlled by the degree of GMA substitution of dextran. (b): The degree of GMA substitution was fixed at 16\%. Measurement of the release rate was carried out at $25^{\circ} \mathrm{C}$ for insulin and $37^{\circ} \mathrm{C}$ for lysozyme. The data shown are the average of three determinations and the error bars represent the standard deviation.

tained lysozyme (data not shown). Apparent release rate constants were calculated from the release profiles according to Eq. 4, and these are shown in Fig. 5. The drug release rate decreased as the degree of GMA substitution increased (Fig. 5(a)), and as the dextran concentration increased (Fig. 5(b)), in a similar way to the diffusion coefficient shown in Fig. 3.

Release profiles of a drug from hydrogels can also be described theoretically by Eq. 5 using the drug diffusion coefficient, $D{ }^{16)}$

$$
\begin{aligned}
& M_{t} / M_{0}=4\left(D t / \pi a^{2}\right)^{1 / 2}-\pi\left(D t / \pi a^{2}\right)-\pi / 3\left(D t / \pi a^{2}\right)^{3 / 2}+4\left(D t / \pi l^{2}\right)^{1 / 2} \\
& -2 a / /\left[8\left(D t / \pi a^{2}\right)-2 \pi\left(D t / \pi a^{2}\right)^{3 / 2}-2 \pi / 3\left(D t / \pi a^{2}\right)^{2}\right]
\end{aligned}
$$

where $M_{t} / M_{0}$ is the fraction of drug released at time $t$, and $a$ and $l$ are the radius and thickness of the hydrogel disk $(3 \mathrm{~mm}$ and $2 \mathrm{~mm}$, respectively, for the hydrogels studied). The diffusion coefficient of insulin in the hydrogels studied was calculated by fitting a series of the $M_{t} / M_{0}$ data shown in Fig. 4 to Eq. 5, using a non-linear least squares program. The diffusion coefficient of lysozyme was also calculated in a similar way to insulin. The diffusion coefficient obtained was compared with that determined by the NMR method. Good agreement was observed between both diffusion coefficients, as shown in Fig. 6. This indicates the usefulness of the NMR method for predicting the release rate of protein and peptide drugs from hydrogels. 


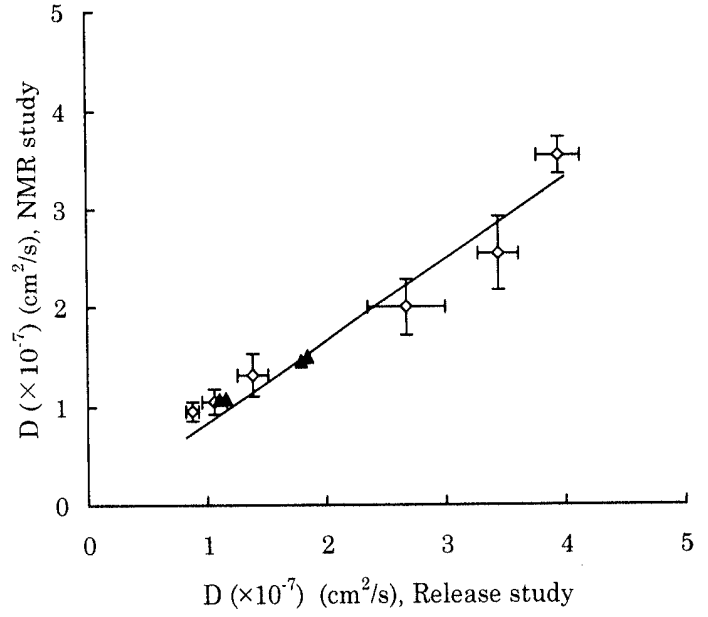

Fig. 6. Comparison of the Diffusion Coefficient Determined by the Pulsed-Field Gradient Stimulated-Echo Method and that Calculated from the Release Profiles

$\diamond$, Insulin $\left(25^{\circ} \mathrm{C}\right) ; \mathbf{\Lambda}$, Lysozyme $\left(37^{\circ} \mathrm{C}\right)$.

In conclusion, the diffusion coefficient of insulin and lysozyme in dextran hydrogels was determined successfully by the stimulated-echo method using a conventional NMR probe, which can impose a magnetic field gradient of 30 Gauss $/ \mathrm{cm}$. This method is applicable to proteins and peptides with a molecular weight of about 10000 or less. The diffusion coefficient obtained by this method can be a measure of drug release from hydrogels.

\section{References}

1) Park K., Shalaby W. S., Park H., "Biodegradable Hydrogels for Drug Delivery Systems," Technimic Publishing, Basel, Switzerland, 1993.

2) Kim S. W., Bae Y. H., Okano T., Pharm. Res., 9, 283-290 (1992).

3) Westrin B. A., Axelsson A., Zacchi G., J. Controlled Rel., 30, 189199 (1994).

4) Gao P., Fagerness P. E., Pharm. Res., 12, 955-964 (1995).

5) Peschier L. J. C., Bouwstra J. A., De Bleyster J., Junginger H. E., Leyte J. C., Biomaterials, 14, 945—952 (1993).

6) Blinc A., Lahajnar G., Blinc R., Zidansek A., Sepe A., Magn. Reson. Med., 14, 105-122(1990).

7) Jones, J. A., Wikins D. K., Smith L. J., Dobson C. M., J. Biomol. NMR, 10, 199-203 (1997).

8) Ilyina E., Roongta V., Pan H., Woodward C., Mayo K. H., Biochemistry, 36(11), 3383-3388 (1997).

9) Dingley A. J., Mackay J. P., Chapman B. E., Morris M. B., Kuchel P. W., Hambly B. D., King G. F., J. Biomol. NMR, 6, 321-328 (1995).

10) Lin M., Larive C. K., Anal. Biochem., 229, 214-220 (1995).

11) Tanner J. E., J. Chem. Phys., 52, 2523-2526 (1970).

12) Hennink W. E., Talsma H., Borchert J. C. H., De Smedt S. C., Demeester J., J. Controlled Rel., 39, 47-55 (1996).

13) Kamath K. R., Park K., Proceed. Intern. Symp. Control. Rel. Bioact. Mater., 19, 42-43 (1992).

14) Aso Y., Yoshioka S., Nakai Y., Kojima S., Radiat. Phys. Chem., accepted, (1997).

15) Higuchi T., J. Pharm. Sci., 52, 1145-1149 (1963).

16) Ritger P. L., Peppas N. A., J. Controlled Rel., 5, 23-36 (1987). 